\title{
Comparison of the safety of brivaracetam at various doses among patients with epilepsy: A network meta-analysis of randomized controlled trials
}

\author{
YING MENG ${ }^{*}$, JIAHUAN WU*, JIANXIN SHI ${ }^{*}$, WENYU WENG and ZHIKUN ZHOU \\ Department of Pharmacology, Guangdong Medical University, Dongguan, Guangdong 523808, P.R. China
}

Received September 28, 2019; Accepted March 10, 2020

DOI: $10.3892 /$ etm.2020.9262

\begin{abstract}
The present study aimed to compare the safety of brivaracetam (BRV) at various doses among patients with epilepsy through a network meta-analysis. Randomized controlled trials (RCTs) were retrieved from different databases, which were then pooled for a network analysis for calculating the odds ratios (ORs), together with the corresponding 95\% confidence intervals (CIs) and surface under the cumulative ranking curve (SUCRA). A total of 9 RCTs were included in the final analysis. Compared with placebo, BRV at a dose of $50 \mathrm{mg}$ daily led to a markedly increased risk of nervous system disorders (OR, 0.62; 95\% CI, 0.43-0.90; $\mathrm{P}=0.01$ ) and evidently increased the risk of psychiatric disorders (OR, 0.16; 95\% CI, 0.04-0.64; $\mathrm{P}=0.022$ ). However, BRV treatment was not associated with a statistically significant change in the prevalence of infectious diseases. SUCRA analysis suggested that treatment with BRV at $50 \mathrm{mg} /$ day posed the highest risk of nervous system disorders and psychiatric disorders compared with placebo or other doses of BRV. In conclusion, BRV treatment at a dose of $50 \mathrm{mg} /$ day may increase the risk of nervous system diseases and psychosis disorders compared with the placebo group. However, more high-quality clinical studies are warranted to validate these results.
\end{abstract}

\section{Introduction}

Epilepsy is a neurologic disorder resulting from abnormal electrical activity of brain neurons, affecting $>70$ million individuals worldwide (1). Multiple factors are involved in the pathogenesis of epilepsy, which reflects the underlying brain dysfunction (2); furthermore, it is difficult to fully explain the disease etiology (3). So far, epilepsy is mainly

Correspondence to: Dr Zhikun Zhou, Department of Pharmacology, Guangdong Medical University, 1 Xincheng Avenue, Songshan Lake, Dongguan, Guangdong 523808, P.R. China

E-mail: zhikunzhou@126.com

${ }^{*}$ Contributed equally

Key words: brivaracetam, epilepsy, safety, network analysis treated by surgical intervention, pharmacotherapy and other approaches (4), including ketogenic dietary treatment $(5,6)$; however, pharmacotherapy remains the dominant therapeutic approach. Effective control over epilepsy may reduce disability and mortality rates (7). As a novel agent to treat epilepsy, brivaracetam (BRV) is a ligand exhibiting high affinity for synaptic vesicle protein $2 \mathrm{~A}$ (SV2A) and selectively combines with SV2A to exert its potent anti-epileptic effects $(8,9)$. In addition, $\mathrm{SV} 2 \mathrm{~A}$ is a protein for regulating synaptic vesicle exocytosis and neurotransmitter release $(10,11)$. With regard to the safety of BRV, numerous studies have suggested that BRV exhibits superior tolerability to other antiepileptic agents (12-19). However, the safety of BRV at various doses has remained controversial, with adverse events including nervous system disorders, psychiatric disorders, infections. Nervous system disorders refer to a class of symptoms associated with the nervous system, including dizziness, headache and hypoaesthesia. Furthermore, psychiatric disorders are defined as symptoms associated with patient consciousness, including anxiety and factitious disorder. Infections are infectious diseases, e.g. urinary tract infection and pneumonia. Therefore, the present study aimed to compare the safety of BRV at various doses among patients with epilepsy by means of a network meta-analysis.

\section{Materials and methods}

Data sources and retrieval. The present network meta-analysis was performed in accordance with the Cochrane Handbook (20). In brief, electronic databases, including Pubmed, Embase and the Cochrane Library, were comprehensively retrieved from inception to June 2019, using the following search terms: ('Brivaracetam' or 'briviact' or 'UCB34714') AND ('epilepsies' OR 'seizure disorder' OR 'awakening epilepsy' OR 'epilepsies, cryptogenic' OR 'aura'). In addition, results from unpublished trials were also retrieved from www.clinicaltrials.gov. Two reviewers (YM and JW) retrieved studies from the databases independently.

Study selection and quality assessment. The above two reviewers were responsible for study selection and quality assessment independently. Any disagreement between them was settled by consensus with a third investigator (JS). Specifically, the study inclusion criteria were as follows: Randomized controlled trails (RCTs); studies in which 
seizure patients (regardless of age) were treated with BRV at various doses $(5,20,50,100$ and $200 \mathrm{mg}$ /day) or placebo; and studies that reported the safety data of interest, including nervous system disorders, psychiatric disorders, infections and infestations. The Cochrane Collaboration tool (21) was used to evaluate the risks of bias among those eligible studies, including selection bias, performance bias, attrition bias, detection bias, reporting bias and other bias.

Data extraction. The following data were extracted from the studies included by two reviewers (YM and JW) independently, including registry number, name of drug, BRV dose, total patient number, number of events in each group, treatment period, drug regimen, mean age, as well as frequencies of nervous system disorders, psychiatric disorders, and infections. In addition, www.clinicaltrials.gov was searched for any unpublished data.

Data analysis. Stata v.12 and NetmetaXL v.1.6.1 software was adopted to perform the present network meta-analysis. Among them, Stata v.12 was used for conventional meta-analysis using the random-effects models, whereas NetmetaXL v.1.6.1 was employed for network meta-analysis using the Bayesian random-effects models to combine the estimates of direct and indirect treatment comparisons. The Mantel-Haenszel $\chi^{2}$ test and I-parameter test were used to assess heterogeneity. Furthermore, heterogeneity was assessed by determining the $\mathrm{I}^{2}$ value and a fixed-effects model was used in the presence of $\mathrm{I}^{2}<50 \%$; otherwise, a random-effects model was employed. Furthermore, the random-effects models were utilized to calculate the odds ratio (OR) and the corresponding 95\% confidence interval (CI). In this analysis, the OR was estimated based on placebo as the control. $\mathrm{P}<0.05$ was considered to indicate statistical significance. For network meta-analysis, we used the Markov Chain Monte Carlo (MCMC) algorithm to produce the result. A total of 2,000 simulations per chain were performed for initial burn-in using the Markov Chain Monte Carlo algorithm, followed by an additional 50,000 simulations, and a total of 3 MCMC chains were adopted. In addition, the goodness of fit was evaluated using the deviance information criterion. Furthermore, the surface under the cumulative ranking curve (SUCRA) was plotted for ranking, where a lower SUCRA value indicated higher risk of adverse events. In addition, the publication bias was assessed using funnel plots and sensitivity analysis was accomplished through omitting one individual study at a time.

\section{Results}

Search results and study characteristics. A total of 378 studies were retrieved after searching the electronic databases Pubmed, Embase, the Cochrane Library and www.clinicaltrials.gov. A total of 9 RCTs including 2,538 patients were selected for the final analysis and all of them were registered at www.clinicaltrials.gov (15-19). Fig. 1 displays the procedure of the literature search. The average age of these participants ranged from 33.2 to 65.6 years and the treatment period ranged from 4 to 17 weeks. Table SI summarizes the characteristics of those 9 RCTs included.
Study evaluation and publication bias. A total of 4 studies, NCT00698581 (15), NCT01261325 (18), NCT00504881 (19) and NCT00699283 had a high risk of bias due to incomplete outcome data and 1 study (NCT03021018) had a high risk of performance bias, while most studies had a low risk of bias. Risk of bias graph and a summary of the included studies are presented in Fig. S1, whilst results of the funnel plots for publication bias are provided in Fig. S2. The results suggested that no publication bias was present for nervous system disorders $(\mathrm{P}=0.175)$, psychiatric disorders $(\mathrm{P}=0.712)$, or infections $(\mathrm{P}=0.283)$.

Conventional meta-analysis of adverse events. Results of conventional meta-analysis are provided in the supplementary material (Table SII). With regard to nervous system disorders, compared with placebo, BRV at the doses of 50 and $200 \mathrm{mg} /$ day was associated with a higher risk (OR, 0.62; 95\% CI, 0.43-0.90; P=0.01; and OR, 0.46; 95\%CI, 0.32-0.65; $\mathrm{P}<0.001$, respectively). BRV at a dose of $50 \mathrm{mg} /$ day was found to be associated with higher risk compared with that in BRV at the dose of $20 \mathrm{mg} /$ day (OR, 0.85; 95\%CI, 0.45-0.95; $\mathrm{P}=0.026$ ). As for psychiatric disorders, BRV at $50 \mathrm{mg} /$ day was associated with a markedly increased risk and the difference was statistically significant compared with placebo (OR, 0.16; 95\% CI, 0.04-0.64; $\mathrm{P}=0.022)$. $\mathrm{BRV}$ at $100 \mathrm{mg} /$ day was demonstrated to be associated with a higher risk compared with that in BRV at $50 \mathrm{mg} /$ day (OR, 5.95; 95\%CI, 1.58-22.38; $\mathrm{P}=0.014)$. In terms of infections, $\mathrm{BRV}$ did not pose a significant risk relative to placebo.

Network meta-analysis of adverse events. In the network maps of nervous system disorders (Fig. 2A), psychiatric disorders (Fig. 2B), and infections (Fig. 2C), the nodes represent the interventions that are compared, the lines stand for the direct comparisons between interventions, the weight of the lines between interventions indicates the number of studies in each comparison and the size of the nodes indicate the actual sample size of each intervention. There were a total of 7 studies, including 29 direct comparisons, reported adverse neurological events (Fig. 2A). A total of 4 studies, including 16 direct comparisons, reported mental illness (Fig. 2B) and 5 studies, including 22 direct comparisons, described the infections (Fig. 2C).

Fig. 3 presents the results of the network meta-analysis. There were no statistically significant differences in nervous system disorders between placebo and BRV at various doses, or among the different doses of BRV (Fig. 3A). With regard to psychiatric disorders (Fig. 3B), BRV at $50 \mathrm{mg} /$ day was associated with a significantly increased risk compared with placebo (OR, 0.09; 95\% Cr.I, 0.01-0.52). Conventional meta-analysis also came to a similar conclusion (Table SII). Furthermore, no significant influences were found with regards to infections (Fig. 3C; Table SII).

Ranking probability. Table I summarizes the results of the SUCRA, which represent the ranking probability of BRV at various doses. In terms of the risk of nervous system disorders, BRV at $50 \mathrm{mg} /$ day was associated with the highest probability of risk, followed by that at $200,100,5$ and $20 \mathrm{mg} /$ day $(0.142$, $0.180,0.485,0.575$ and 0.739 , respectively). As for the risk of 


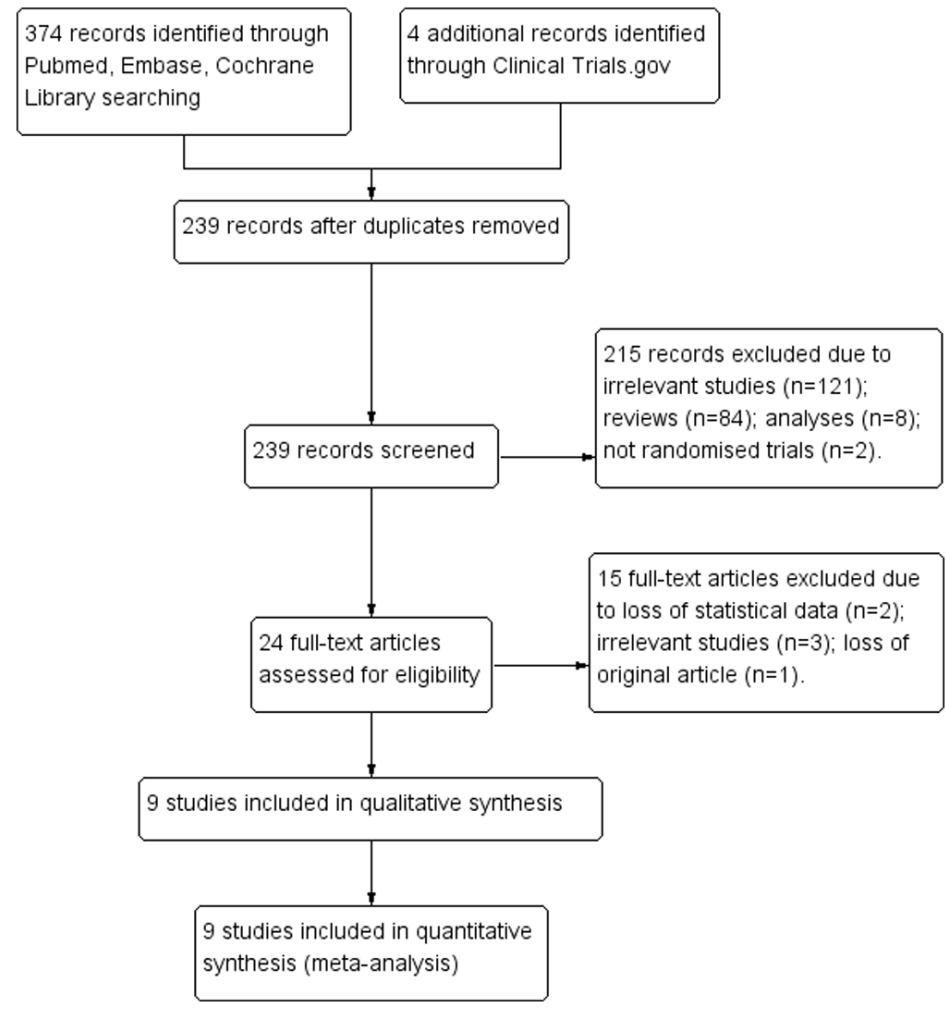

Figure 1. Flow chart depicting the search and selection strategy of studies for the present network meta-analysis.

A
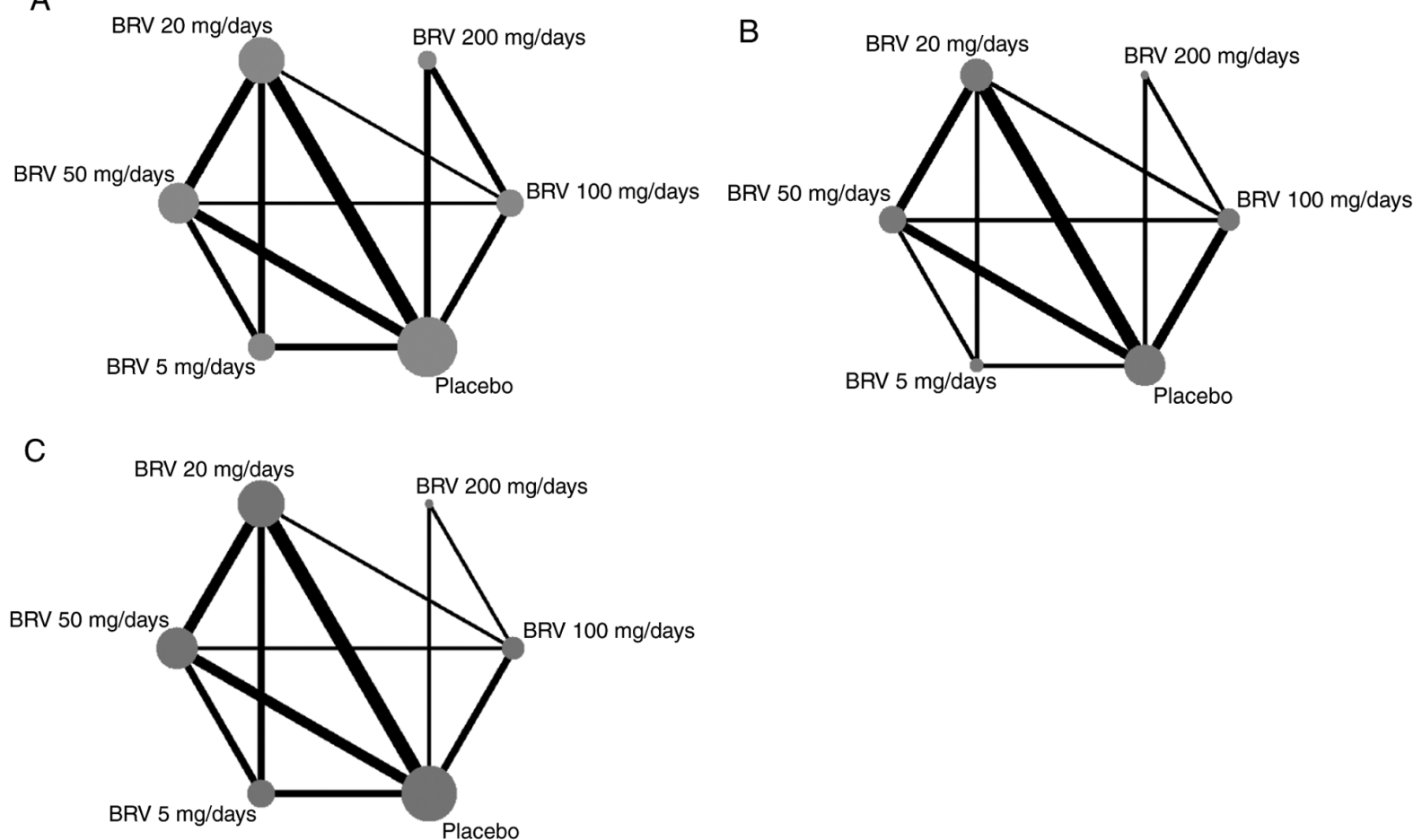

Figure 2. The network plot of nervous system disorders, psychiatric disorders and infections associated with BRV treatment at different doses. (A) nervous system disorders, (B) psychiatric disorders and (C) infections. The nodes represent the interventions that are compared, the lines stand for the direct comparisons between interventions, the weight of lines between interventions indicates the number of studies in each comparison and the size of the nodes indicate the actual sample size of each intervention. BRV, brivaracetam.

psychiatric disorders, BRV at $50 \mathrm{mg} /$ day had the highest-ranking probability, followed by that at $100,20,5$ and $200 \mathrm{mg} /$ day $(0.037,0.367,0.508,0.590$ and 0.765 , respectively). As for the risk of infections, BRV at $5 \mathrm{mg} /$ day had the highest-ranking probability, followed by that at $20,100,50$ and $200 \mathrm{mg} / \mathrm{day}$ $(0.109,0.319,0.499,0.771$ and 0.892 , respectively). The data 
A

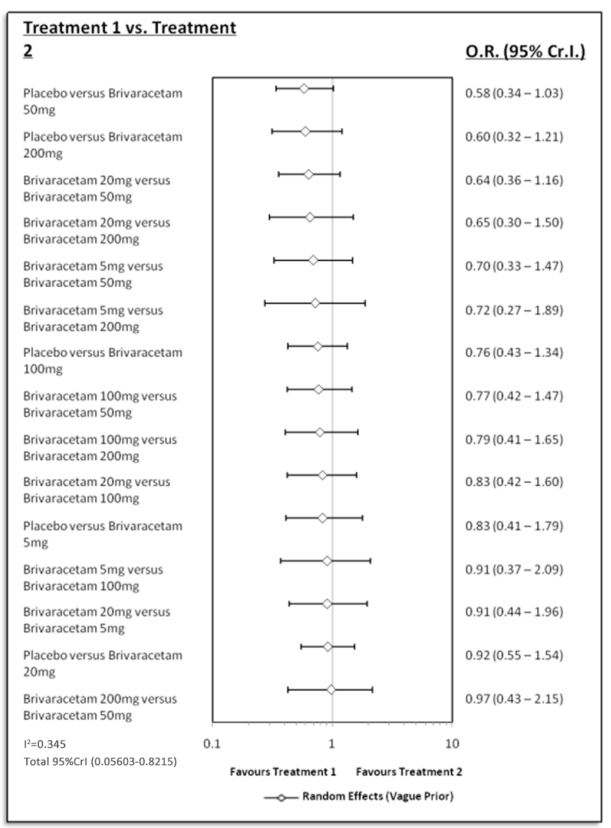

C

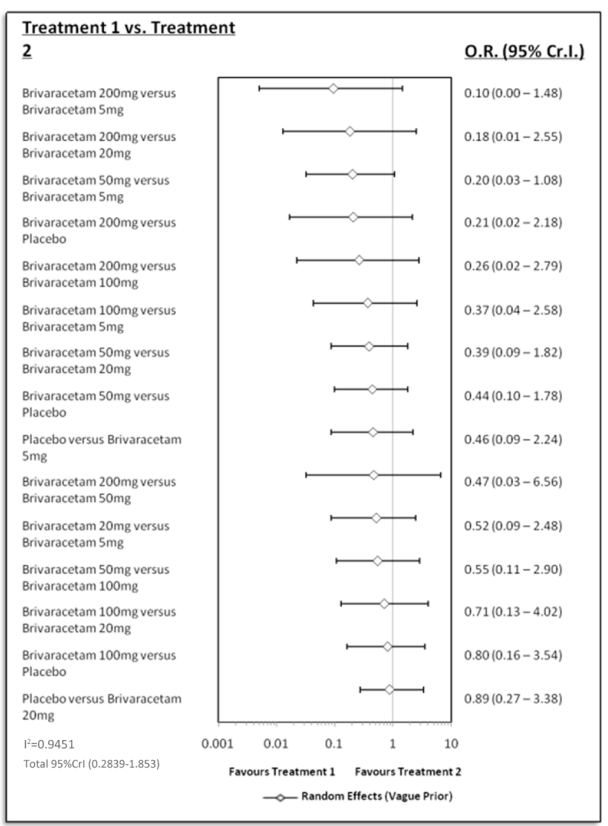

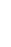

B

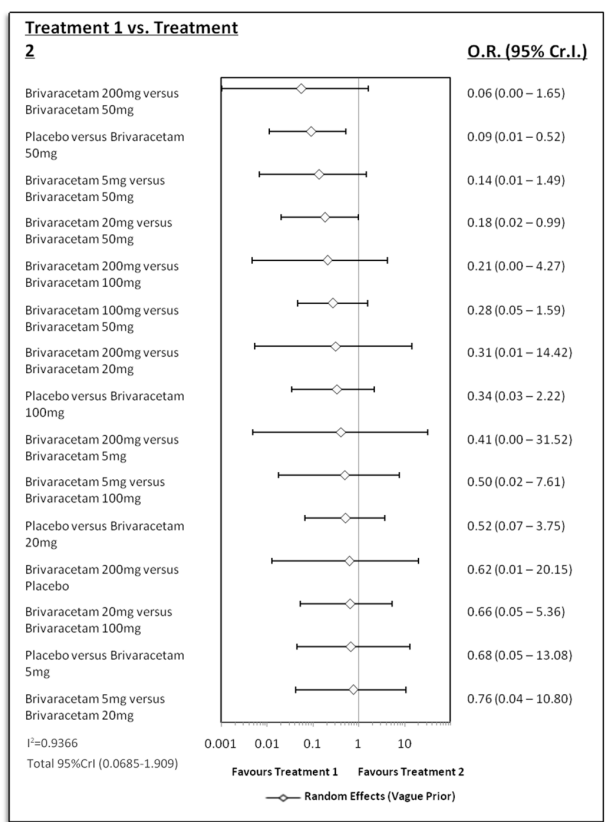

Figure 3. Results of the network meta-analysis. (A) nervous system disorders, (B) psychiatric disorders and (C) infections. O.R., odds ratio; Cr.I, confidence interval.

show that BRV at $50 \mathrm{mg} /$ day represented the highest risk of nervous system disorders and psychiatric disorders since the SUCRA value was found to be the lowest.

Sensitivity analysis. Sensitivity analysis was also performed through omitting one individual study at a time (Fig. S3), which revealed that the results were not obviously changed when any of the other studies was removed.

\section{Discussion}

Epilepsy is a frequent serious brain disease. In 1/4 of patients, antiepileptic drugs cannot be used to control epilepsy, which severely affects the quality of life of those patients (22). Adverse events in the nervous system occur more frequently during the treatment for patients (18). Psychiatric disorders and infectious diseases are also common, as identified in certain clinical trials (10). However, the mechanism of these comorbidities has remained elusive. The present network meta-analysis was performed based on 9 RCTs involving 2,538 participants. The major outcomes of the network meta-analysis suggested the following: i) BRV at $50 \mathrm{mg} /$ day resulted in a statistically significant increase in nervous system disorders compared with placebo; ii) BRV at $50 \mathrm{mg} /$ day led to a statistically significant increase in psychiatric disorders compared with placebo and it also had the highest risk; and iii) BRV was not associated with a statistically significant difference in infectious diseases compared with placebo. 
Table I. SUCRA values for nervous system disorders, psychiatric disorders, infections.

\begin{tabular}{lccc}
\hline & \multicolumn{3}{c}{ Adverse events } \\
\cline { 2 - 4 } $\begin{array}{l}\text { Dose of } \\
\text { BRV (mg/day) }\end{array}$ & $\begin{array}{c}\text { Nervous system } \\
\text { disorders }\end{array}$ & $\begin{array}{c}\text { Psychiatric } \\
\text { disorders }\end{array}$ & Infections \\
\hline 5 & 0.575 & 0.590 & 0.109 \\
20 & 0.739 & 0.508 & 0.319 \\
50 & 0.142 & 0.037 & 0.771 \\
100 & 0.485 & 0.367 & 0.499 \\
200 & 0.180 & 0.765 & 0.892 \\
Placebo & 0.875 & 0.732 & 0.408 \\
\hline
\end{tabular}

The values range between 0 and 1 where 1 is designated as the maximum value. A lower SUCRA value indicated higher occurrence of adverse events. BRV, brivaracetam; SUCRA, surface under the cumulative ranking curve.

Nervous system disorders are a class of relatively common adverse events. The results of the conventional meta-analysis performed in the present study suggested that BRV at $50 \mathrm{mg} /$ day markedly increased the risk of such complications. Furthermore, the results of the network meta-analysis demonstrated that BRV at $50 \mathrm{mg} /$ day was associated with the lowest SUCRA value of 0.142 , indicating that, compared with other doses of BRV, BRV at $50 \mathrm{mg} / \mathrm{day}$ was associated with the highest risk of such adverse effects. However, it is difficult to clearly explain the exact mechanisms of this phenomenon. The results of the present network meta-analysis may provide certain guidance for clinical practice, e.g. more attention should be paid to nervous system diseases when treating epilepsy patients with BRV at a dose of $50 \mathrm{mg} /$ day or higher and disease deterioration should also be avoided.

Mental disorders occurring during the course of epilepsy have attracted the attention of clinicians and researchers, and they represent a serious comorbidity in epilepsy (23). The association between epilepsy and psychiatric disorders is more complex than expected, as elaborated in a recent review (23). These comorbidities were previously thought to be adverse drug reactions, but they are currently considered to be part of epilepsy, which may be attributed to potential disorders of the neural network (24). As suggested by the results of the conventional meta-analysis performed in the present study, BRV at a dose of $50 \mathrm{mg} /$ day markedly increased the risk of psychotic disorders compared with placebo. Furthermore, network meta-analysis suggested that, compared with other doses, BRV at the dose of $50 \mathrm{mg} /$ day led to the lowest SUCRA value (0.037), indicating that it was linked to the highest risk of psychotic disorders. Typically, the risk of such an adverse reaction was detected at the BRV dose of $50 \mathrm{mg} / \mathrm{day}$. Therefore, clinicians should pay more attention to the mental conditions of epilepsy patients treated with BRV at a dose of $50 \mathrm{mg} /$ day or higher. BRV should be considered for continued use in the presence of a BRV-induced psychiatric disorder.

Levetiracetam, an analogue of BRV, has the same mechanism of action as BRV, and these two antiepileptic drugs exert their anti-epileptic effects through binding to SV2A (8). With regard to the infectious disease, a meta-analysis suggests that application of BRV alone does not increase the risk of infectious disease (25), which is consistent with the present results. That study also pointed out that levetiracetam is associated with a significantly higher risk of infectious diseases than BRV (25). In addition, another study suggests that SV2A is expressed in $\mathrm{CD} 8^{+} \mathrm{T}$ lymphocytes and inhibiting SV2A affects the function of $\mathrm{CD}^{+} \mathrm{T}$ lymphocytes, which causes an increased risk of infectious diseases (26). However, it is unable to explain why BRV makes no difference to the increased risk of infectious diseases. After all, they share the same mechanism of action. Conversely, other studies suggested that levetiracetam exerts its anti-inflammatory activity through regulating inflammatory factors $(27,28)$. BRV may have the same anti-inflammatory and pro-inflammatory effects as those of levetiracetam, of which the former exerts a more potent role than the latter, and BRV is suggested not to be associated with an increased risk of infectious disease. However, such conjecture has not been accurately verified so far, and these contradictory views have added to the complexity in interpreting the results. In fact, the statistical results of the present study involved all infectious diseases rather than a specific one. However, the studies with a small sample size included in the present meta-analysis only provided short-term data, which may limit the present results. The above aspects may account for the differences in results between BRV and levetiracetam.

Certain limitations should be noted regarding the present analysis. Firstly, only 9 RCTs were included for final analysis and such a small sample size may affect the results of the analysis. Furthermore, the treatment duration in these studies was relatively short, and therefore, the present results do not represent those from long-term clinical trials for epilepsy patients who took medication for a long time. Finally, the present network meta-analysis was performed in accordance with the Cochrane Handbook instead of PRISMA guidelines (29), which is beneficial to improve the quality of the analysis. Therefore, more high-quality clinical studies are still warranted to validate these results.

To sum up, the present network meta-analysis may provide certain guidance for clinical practice. Specifically, more attention should be paid to the risks of nervous system disease and psychotic disorders when a patient is treated with BRV at a dose of $50 \mathrm{mg} /$ day or higher. Actually, the results obtained with healthy participants demonstrate that these adverse reactions are mild to moderate and disappear within 1 day (11). However, such results are based on healthy subjects and cannot be extrapolated to all patients, particularly those at a high risk of nervous system disease or psychosis disorder. Thus, clinicians should pay more attention to the mental conditions of patients.

\section{Acknowledgements}

Not applicable.

\section{Funding}

The present study was supported by the National Natural Science Foundation of China (grant no. 81774344). 


\section{Availability of data and materials}

All data generated or analyzed during this study are included in this published article.

\section{Authors' contributions}

YM and ZZ deigned the current study. YM, JW and JS were responsible for study retrieval. YM, WW and JS contributed to data collection and analysis. YM and JW wrote the manuscript. All authors read and approved the final manuscript.

\section{Ethics approval and consent to participate}

Not applicable.

\section{Patient consent for publication}

Not applicable.

\section{Competing interests}

The authors declare that they have no competing interests.

\section{References}

1. Ngugi AK, Bottomley C, Kleinschmidt I, Sander JW and Newton CR: Estimation of the burden of active and life-time epilepsy: A meta-analytic approach. Epilepsia 51: 883-890, 2010

2. Shorvon SD, Andermann F and Guerrini R: The causes of epilepsy. Cambridge University Press, Cambridge, 2011.

3. Manford M: Recent advances in epilepsy. J Neurol 264: 1811-1824, 2017.

4. Liu G, Slater N and Perkins A: Epilepsy: Treatment options. Am Fam Physician 96: 87-96, 2017.

5. Felton EA and Cervenka MC: Dietary therapy is the best option for refractory nonsurgical epilepsy. Epilepsia 56: 1325-1329, 2015.

6. D'Andrea Meira I, Romão TT, Pires do Prado HJ, Krüger LT, Pires MEP and da Conceição PO: Ketogenic diet and epilepsy: What we know so far. Front Neurosci 13: 5, 2019.

7. Thijs RD, Surges R, O'Brien TJ and Sander JW: Epilepsy in adults. Lancet 393: 689-701, 2019.

8. Klitgaard H, Matagne A, Nicolas JM, Gillard M, Lamberty Y, De Ryck M, Kaminski RM, Leclercq K, Niespodziany I, Wolff C, et al: Brivaracetam: Rationale for discovery and preclinical profile of a selective SV2A ligand for epilepsy treatment. Epilepsia 57: 538-548, 2016.

9. Nicolas JM, Hannestad J, Holden D, Kervyn S, Nabulsi N and Tytgat D: Brivaracetam, a selective high-affinity synaptic vesicle protein 2A (SV2A) ligand with preclinical evidence of high brain permeability and fast onset of action. Epilepsia 57: 201-209, 2016

10. Mula M: Brivaracetam for the treatment of epilepsy in adults. Expert Rev Neurother 14: 361-365, 2014.

11. Malawska B and Kulig K: Brivaracetam: A new drug in development for epilepsy and neuropathic pain. Expert Opin Investig Drugs 17: 361-369, 2008.

12. Li-Na Z, Deng C, Hai-Jiao W, Da X, Ge T and Ling L: Indirect comparison of third-generation antiepileptic drugs as adjunctive treatment for uncontrolled focal epilepsy. Epilepsy Res 139: $60-72,2018$.
13. Zhu LN, Chen D, Xu D, Tan G, Wang HJ and Liu L: Newer antiepileptic drugs compared to levetiracetam as adjunctive treatments for uncontrolled focal epilepsy: An indirect comparison. Seizure 51: 121-132, 2017

14. Zaccara G, Giovannelli F, Giorgi FS, Franco V, Gasparini S and Benedetto U: Tolerability of new antiepileptic drugs: A network meta-analysis. Eur J Clin Pharmacol 73: 811-817, 2017.

15. Arnold S, Badalamenti V, Diaz A, Gasalla T, McShea C and Whitesides J: Conversion to brivaracetam monotherapy for the treatment of patients with focal seizures: Two double-blind, randomized, multicenter, historical control, phase III studies. Epilepsy Res 141: 73-82, 2018.

16. Biton V, Berkovic SF, Abou-Khalil B, Sperling MR, Johnson ME and $\mathrm{Lu} \mathrm{S}$ : Brivaracetam as adjunctive treatment for uncontrolled partial epilepsy in adults: A phase III randomized, double-blind, placebo-controlled trial. Epilepsia 55: 57-66, 2014.

17. French JA, Costantini C, Brodsky A and von Rosenstiel P: Adjunctive brivaracetam for refractory partial-onset seizures: A randomized, controlled trial. Neurology 75: 519-525, 2010.

18. Klein P, Schiemann J, Sperling MR, Whitesides J, Liang W and Stalvey T: A randomized, double-blind, placebo-controlled, multicenter, parallel-group study to evaluate the efficacy and safety of adjunctive brivaracetam in adult patients with uncontrolled partial-onset seizures. Epilepsia 56: 1890-1898, 2015.

19. Kwan P, Trinka E, Van Paesschen W, Rektor I, Johnson ME and $\mathrm{Lu} \mathrm{S}$ : Adjunctive brivaracetam for uncontrolled focal and generalized epilepsies: Results of a phase III, double-blind, randomized, placebo-controlled, flexible-dose trial. Epilepsia 55: 38-46, 2014.

20. Higgins JPT, Thomas J, Chandler J, Cumpston M, Li T, Page MJ and Welch VA (editors): Cochrane Handbook for Systematic Reviews of Interventions version 6.0 (updated July 2019). Cochrane, 2019. htttps://www.training.cochrane.org/handbook.

21. Julian PTH, Douglas GA, Peter CG, Peter J, David M, Andrew DO, Jelena S, Kenneth FS and Laura W: The Cochrane Collaboration's tool for assessing risk of bias in randomised trials. British Medical Journal 343: 889-893, 2011.

22. Kwan P, Schachter SC and Brodie MJ: Drug-resistant epilepsy. N Eng J Med 365: 919-926, 2011.

23. Agrawal N and Mula M: Treatment of psychoses in patients with epilepsy: An update. Ther Adv Psychopharmacol 9: $2045125319862968,2019$.

24. Stafstrom CE and Carmant L: Seizures and epilepsy: An overview for neuroscientists. Cold Spring Harb Perspect Med 5: a022426, 2015.

25. Zaccara G, Giovannelli F, Giorgi FS, Franco V, Gasparini S and Tacconi FM: Do antiepileptic drugs increase the risk of infectious diseases? A meta-analysis of placebo-controlled studies. Br J Clin Pharmacol 83: 1873-1879, 2017.

26. Nowak M, Bauer S, Schlegel K, Stei S, Allenhöfer L, Waschbisch A, Tackenberg B, Höllerhage M, Höglinger GU, Wegner S, et al: Levetiracetam but not valproate inhibits function of $\mathrm{CD}^{+} \mathrm{T}$ lymphocytes. Seizure 22: 462-466, 2013.

27. Erbaş O, Yeniel AÖ, Akdemir A, Ergenoğlu AM, Yilmaz M, Taskiran D and Peker G: The beneficial effects of levetiracetam on polyneuropathy in the early stage of sepsis in rats: Electrophysiological and biochemical evidence. J Invest Surg 26: 312-318, 2013.

28. Itoh K, Ishihara Y, Komori R, Nochi H, Taniguchi R, Chiba Y, Ueno M, Takata-Tsuji F, Dohgu S and Kataoka Y: Levetiracetam treatment influences blood-brain barrier failure associated with angiogenesis and inflammatory responses in the acute phase of epileptogenesis in post-status epilepticus mice. Brain Res 1652: 1-13, 2016.

29. Selçuk AA: A guide for systematic reviews: PRISMA. Turk Arch Otorhinolaryngol 57: 57-58, 2019. 